\title{
Zinc Modulates Mitogenic Responses of Human Lymphocytes by Affecting Structures Influenced by Cytochalasin $\mathbf{B}^{1}$
}

\author{
K. Murali Krishna Rao and Stanley A. Schwartz \\ Department of Pediatrics, The University of Michigan, Ann Arbor, Michigan 48109
}

Received November 23, 1979

\begin{abstract}
We have previously reported that zinc affects the mitogenic process in an agedependent fashion when added to lectin-stimulated lymphocytes. The present studies demonstrate an additional selective effect of zinc when added to Con A-activated lymphocytes plus pharmacologic agents known to affect the cytoskeleton or the permeability of the plasma membrane. Specifically, addition of zinc did not modulate the dosedependent activities of $\mathrm{Ca}^{2+}$ ionophore A23187, nigericin, or colchicine demonstrating that the immunologic effects of zinc do not involve modification of $\mathrm{Ca}^{2+}$ or $\mathrm{K}^{+}$fluxes, or microtubule formation. By contrast, a consistent and reproducible shift in the cytochalasin B dose-response curve of lymphocytes stimulated by Con A was observed when zinc was added. These data indicate that the effect of zinc on immune functions may be operating through cytochalasin B-sensitive subcellular structures, most likely microfilaments.
\end{abstract}

\section{INTRODUCTION}

Zinc plays an important role in immune function. Zinc deficiency in animals is associated with abnormal development of the thymus in cattle (1), the lymphoid system in mice (2), and defective immune function in animals (3-5). Abnormal zinc metabolism is a feature of the syndrome, acrodermatitis enteropathica, a disease associated with T-cell functional abnormalities $(6,7)$. Administration of zinc sulphate alleviates the clinical symptoms in this condition (8).

Zinc can act as a mitogen when added directly to lymphocyte cultures $(9,10)$. Recently we demonstrated that addition of zinc to lectin-stimulated lymphocyte cultures alters mitogenic responses in an age-dependent fashion (11). Lymphocytes from young adults show either enhancement or no change in the mitogenic response on addition of zinc, whereas lymphocytes from healthy aged individuals show suppressed mitogenic responses in zinc-supplemented cultures. The present paper describes experiments performed to delineate the mechanism of the zinc effect on lectin-induced mitogenic responses.

The rationale for the experiments described below is based on the following theoretical model. For the sake of clarity we shall keep the model very simple. The addition of concanavalin $\mathrm{A}$ (Con $\mathrm{A}$ ) to cultures of lymphocytes initiates a series of events ultimately leading to DNA synthesis (Fig. 1). A number of agents can modulate the mitogenic process induced by $\mathrm{Con} A$. These include $\mathrm{Ca}^{2+}$

\footnotetext{
${ }^{1}$ This work was presented at the Mid-West Autumn Immunology Conference, Detroit, Mich.,
} November 5, 1979. 


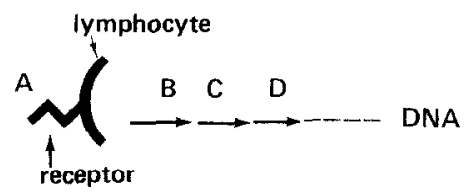

FIG. 1. Theoretical model for transmission of a stimulus from the lymphocyte surface to the nucleus following lectin binding.

ionophore A23187 (12), the $\mathrm{K}^{+}$ionophore, nigericin (13), colchicine, a microtubule-modulating agent $(14)$, and cytochalasin $\mathrm{B}(\mathrm{CB})(15,16)$ which appears to affect the function of microfilaments (17). All these compounds inhibit mitogenic responses at higher concentrations and the inhibitory effect is lost as the concentration of these agents is gradually lowered. This permits one to obtain dose-response curves with respect to mitogenic responses when lymphocytes are stimulated with Con $A$ in the presence of various amounts of these pharmacologic agents. Based on their vastly different biochemical effects, it is reasonable to assume that each of these agents acts at a different site in the sequence of events leading to DNA synthesis, namely A, B, C, D, etc. (Fig. 1). As the mechanisms of action of these compounds are well established, their ability to modify Con Astimulated mitogenic responses can be used advantageously to elucidate the mechanism of another substance which also is known to affect the lectin-induced mitogenic process.

In order to determine the site of action of an agent whose mechanism of action is unknown, the following approach is adopted: lymphocytes are stimulated with Con A to initiate DNA synthesis and a dose-response curve is generated by adding various amounts of a second agent whose site of action is already known (e.g., site B, Fig. 1). The addition of a constant amount of a third compound whose site of action is unknown could lead to one of two possible effects. If the site of action of agent 3 is the same as agent 2 , the net result would be equivalent to a constant increment (or decrement, if agent 3 is antagonistic) in the dose of agent 2. This would lead to a shift in the dose-response curve of agent 2 , and the direction of the shift depends upon whether agent 3 is synergistic or antagonistic to agent 2. On the other hand if the site of action of agent 3 is different from agent 2 , there would not be a significant shift in the dose-response curve. Further, changes in the magnitude of the response without a shift in the curve would indicate a different site of action. Based on this simple model, we hypothesize that if the mechanism of action of two agents is similar there will be a shift in the dose-response curve and the lack of a shift indicates dissimilar mechanisms of action. This model can easily be expanded to fit more complex situations where an agent has more than one site of action. In that event a shift would be expected to occur only when two agents have a common site of action involving a rate-regulating step. The experimental results presented below show that this approach is capable of yielding informative results.

\section{MATERIALS AND METHODS}

Donors. Blood from young (age range: 20 to 35 years) healthy volunteer donors 
was freshly obtained from a peripheral vein. All donors gave informed consent consistent with the policies of The University of Michigan and Department of Health, Education and Welfare.

Isolation of lymphocytes. Peripheral blood lymphocytes (PBL) were prepared from fresh heparinized $(20 \mathrm{U} / \mathrm{ml})$ blood by a modification of the density gradient technique of Böyum (18). The mononuclear cell band was harvested and washed three times with $\mathrm{Ca}^{2+}$ - and $\mathrm{Mg}^{2+}$-free Hanks' balanced salt solution (Grand Island Biological Co., Grand Island, N.Y.) and resuspended in RPMI 1640 medium (Grand Island Biological Co.) containing $10 \%$ heat-inactivated bovine calf serum, $80 \mu \mathrm{g}$ gentamicin/ml (Schering Corp., Kenilworth, N.J.), and $300 \mu \mathrm{g}$ of fresh glutamine/ml.

Cell cultures. Cells were cultured in round-bottom microtiter plates at a concentration of $5 \times 10^{4}$ cells in $200 \mu \mathrm{l}$. Mitogens, drugs, and zinc chloride were added to the wells at appropriate concentrations and the final volume of all cultures was adjusted to $240 \mu \mathrm{l}$. The cells were incubated for $72 \mathrm{hr}$ at $37^{\circ} \mathrm{C}$ in an atmosphere consisting of $5 \% \mathrm{CO}_{2}$ and $95 \%$ air. At $72 \mathrm{hr}, 2 \mu \mathrm{Ci}$ of [methyl- $\left.{ }^{3} \mathrm{H}\right]$-thymidine, specific activity of $6.7 \mathrm{Ci} / \mathrm{mmol}\left(\left[{ }^{3} \mathrm{H}\right]\right.$-thymidine, New England Nuclear, Boston, Mass.), was added to each well and the cultures were incubated for an additional $18 \mathrm{hr}$. The cells were harvested by an automatic cell harvester (Flow Laboratories, Rockville, Md.) and incorporation of $\left[{ }^{3} \mathrm{H}\right]$-thymidine was measured by liquid scintillation spectroscopy.

Drugs. Ionophore A23187 and nigericin were gifts from Eli Lilly Co., Indianapolis, Indiana. Cytochalasin B and colchicine were obtained from Sigma Chemical Co., St. Louis, Missouri. Ionophore A23187, nigericin, and CB were dissolved in absolute ethanol at a concentration of $2 \times 10^{-3}, 1 \times 10^{-3}$, and $2 \times 10^{-2}$ $M$, respectively and stored at $4^{\circ} \mathrm{C}$. Appropriate dilutions were made up in RPMI. Colchicine was dissolved in RPMI and stored frozen. $\mathrm{ZnCl}_{2}$ was made up in sterile distilled water at a concentration of $10^{-1} M$ and appropriate dilutions were made with RPMI.

\section{RESULTS}

Experiments with ionophore A23187. Figure 2 shows the $\mathrm{Ca}^{2+}$ ionophore A23187 dose-response curves of lymphocyte responses obtained with a single mitogen concentration $(10 \mu \mathrm{g} / \mathrm{ml}$ of Con A) with the ionophore alone and in the presence of $5 \times 10^{-5} M$ and $1 \times 10^{-4} M$ zinc. Addition of zinc did not cause any significant shift in the dose - response curve compared to ionophore A23187 alone. Table 1 shows a checkerboard experiment where the effect of zinc $\left(1 \times 10^{-4} \mathrm{M}\right)$ on ionophore A23187 dose responses at three different Con A concentrations was examined. The data show that the addition of zinc did not bring about a shift in dose responses at any mitogen concentration tested.

Experiments with nigericin. Figure 3 shows experiments similar to the above, with nigericin. No significant shift in nigericin dose-response curve was observed at two concentrations of zinc.

Experiments with colchicine. Figure 4 shows Con A-stimulated dose-response curves to colchicine with and without zinc. Once again no shift in the dose-response curve was obtained. Table 2 shows another checkerboard experiment using 


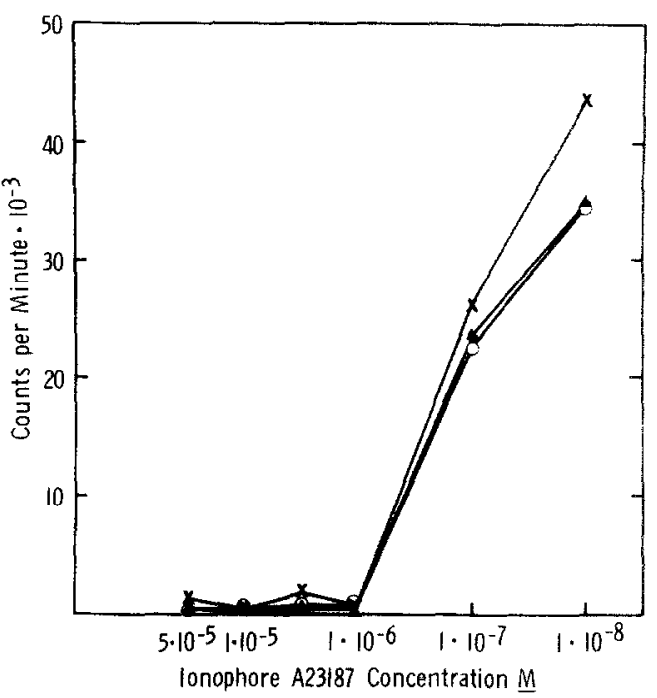

FIG. 2. Effecl of zinc on the jonophore A23187 dose-response curve. $x$, Con $A(10 \mu \mathrm{g} / \mathrm{ml}): O, C o n$ $\mathrm{A}(10 \mu \mathrm{g} / \mathrm{ml})+\mathrm{Zn} 5 \times 10^{-5} M: \Delta$, Con $\mathrm{A}(10 \mu \mathrm{g} / \mathrm{ml})+\mathrm{Zn} 1 \times 10^{-4} M$.

three mitogen concentrations. It can be seen that the addition of zinc fails to bring about a shift in the dose-response curve at all three mitogen concentrations tested.

Experiments with cytochalasin $B$. In contrast to the above results, Fig. 5 demonstrates a clear shift in the dose-response curve of $C B$ stimulated with Con $A$ $(10 \mu \mathrm{g} / \mathrm{ml})$ upon the addition of zinc. The shift in the curve is best seen only at optimal concentrations of the mitogen. This is illustrated in Table 3. As seen in the last row of Table 3, the optimal mitogenic dose of Con A alone was $15 \mu \mathrm{g} / \mathrm{ml}$ for this individual donor. A comparison of the CB dose-response curve at the optimal Con A concentration for this donor $(15 \mu \mathrm{g} / \mathrm{ml})$ with and without zinc reveals that in the presence of zinc the maximal response occurs at a lower concentration of cytochalasin $\mathrm{B}, 10^{-8} M$, instead of at $10^{-7} M$ with CB alone. Thus, this shift in maximal mitogenic response to a lower concentration of $C B$ upon addition of zinc to the system reflects a synergistic effect between zinc and CB as detailed in our theoretical model.

\section{DISCUSSION}

Our previous studies showed that zinc modulates lectin-induced mitogenic responses of human lymphocytes; further the effect was age related (11). The optimum results were observed at a concentration of $1 \times 10^{-4} M$ zinc chloride. Therefore, this particular concentration was chosen for most of the experiments in this study. Employing various concentrations of the mitogen $(5,10$, and $15 \mu \mathrm{g} / \mathrm{ml}$ of Con $\mathrm{A})$ and two concentrations of zinc $\left(5 \times 10^{-5}\right.$ and $\left.1 \times 10^{-4} \mathrm{M}\right)$ we did not observe any shifts in the dose-response curves obtained with potassium ionophore, nigericin, calcium ionophore A23187, and a microtubule-modulating agent colchicine. In contrast, a clear shift in the dose-response curve to $C B$ was observed in the presence of zinc in four of seven experiments employing a single 


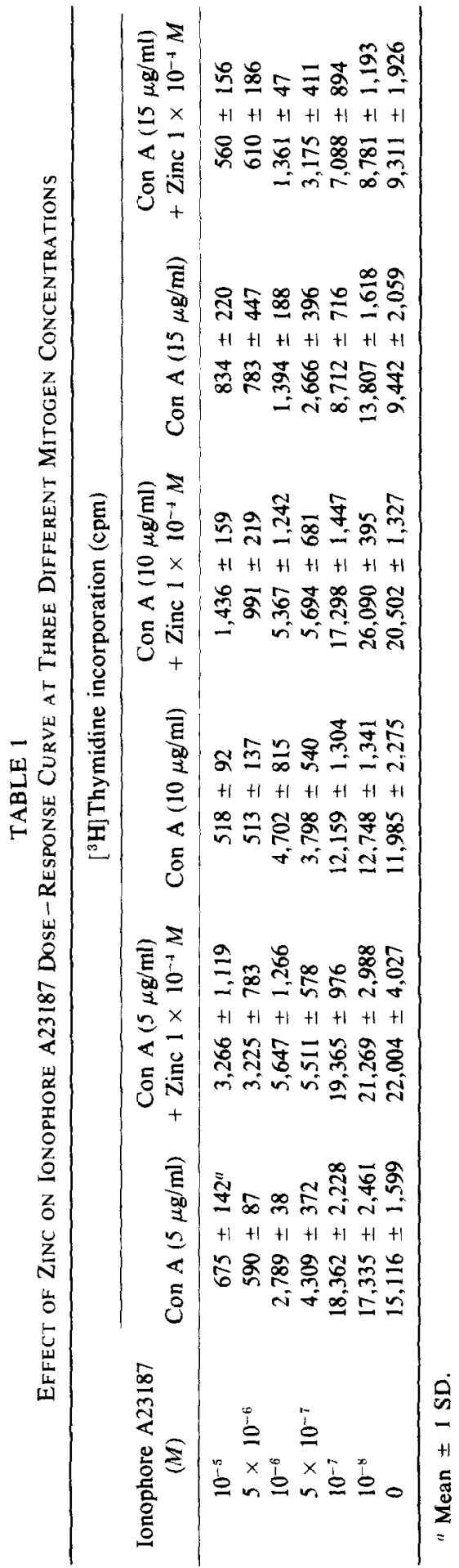




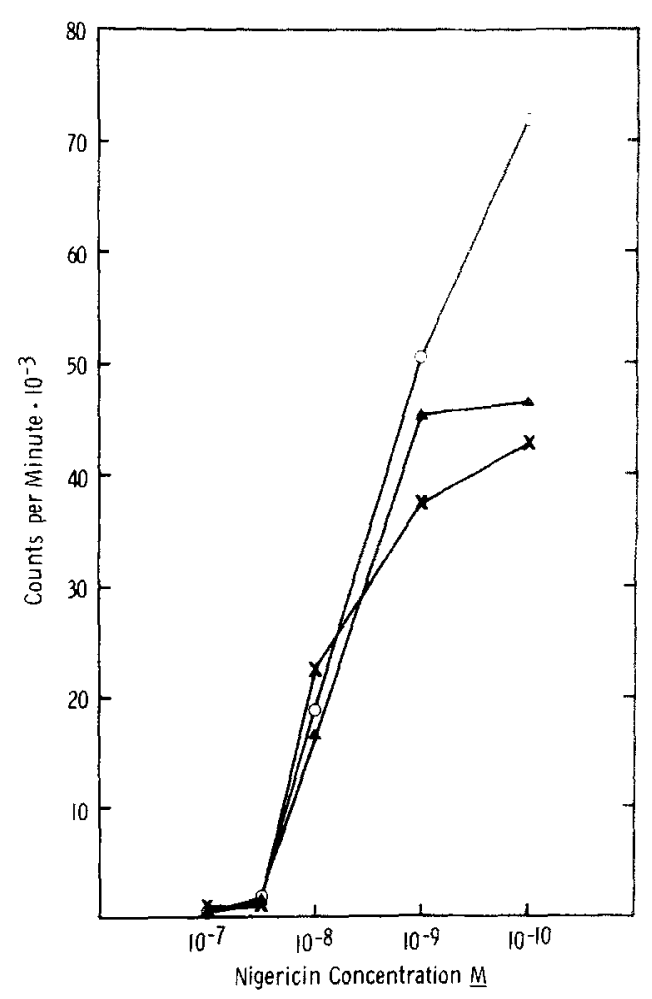

Fig. 3. Effect of zinc on the nigericin dose-response curve. $x$, Con A $(10 \mu \mathrm{g} / \mathrm{ml}) ; 0$, Con A (10 $\mu \mathrm{g} / \mathrm{ml})+\mathrm{Zn} 5 \times 10^{-5} M: \Delta$, Con A $(10 \mu \mathrm{g} / \mathrm{ml})+\mathrm{Zn} 1 \times 10^{-4} M$.

mitogen concentration $(10 \mu \mathrm{g} / \mathrm{ml}$ Con $\mathrm{A})$. In previous studies we found that 10 $\mu \mathrm{g} / \mathrm{ml}$ of Con A was an optimal dose for lymphocytes obtained from a majority of individuals $(11,19)$. Further, under the culture conditions described, optimal mitogenic responses generally fall within a range of $5-15 \mu \mathrm{g} / \mathrm{ml}$ of Con $\mathrm{A}$. Employing three concentrations of Con $\mathrm{A}(5,10$, and $15 \mu \mathrm{g} / \mathrm{ml})$ we observed that the shift in the blast transformation response to $C B$ was observed best only at optimal mitogenic concentrations of Con A. This is consistent with our earlier finding that optimal modulation of the mitogenic response of lymphocytes to zinc occurred usually at the maximal mitogenic responses (11). Thus, it is essential to use more than one concentration of mitogen to study the effects of pharmacologic agents on blatsogenic responses. In contrast to the above results with a single Con A concentration $(10 \mu \mathrm{g} / \mathrm{ml})$ employing three doses of Con $A$ we observed a shift in the Con A-stimulated CB dose-response curve in 10 of 13 experiments.

On the basis of these experimental results we conclude that the effects of zinc on lectin-induced mitogenic responses are not exerted through alterations in $\mathrm{Ca}^{2+}$ or $\mathrm{K}^{+}$fluxes or by alterations in microtubular function. The clear shift in the $\mathrm{CB}$ dose-response curves suggests that zinc might be acting on structures which are influenced by CB.

Existing knowledge of the effect of $\mathrm{CB}$ on subcellular elements is controversial. Compelling evidence has been presented to emphasize that $\mathrm{CB}$ acts specifically 


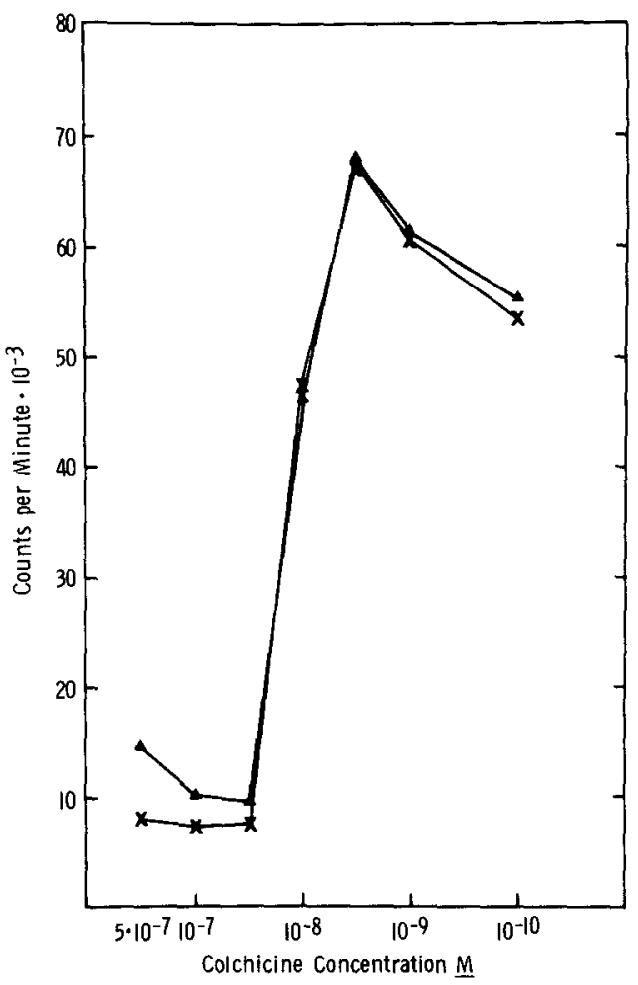

Fig. 4. Effect of zinc on the colchicine dose-response curve. $\mathrm{x}$, Con A $(10 \mu \mathrm{g} / \mathrm{ml}) ; \boldsymbol{\Lambda}$, Con A (10 $\mu \mathrm{g} / \mathrm{ml})+\mathrm{Zn} 1 \times 10^{-4} M$.

through microfilaments (17). Equally persuading contradictions have been cited to argue that such a conclusion is premature (20). However, using rabbit pulmonary macrophages it was demonstrated that low concentrations of $C B$ reversibly inhibit the temperature-dependent gelation of actin by actin-binding protein (21). At present it appears reasonable to assume that $\mathrm{CB}$ is exerting its effects through microfilaments, actin, or similar structures. In addition to its effects on microfilaments, $\mathrm{CB}$ causes an increase in $\mathrm{Ca}^{2+}$ uptake in lymphocytes treated with mitogens (22). However, the inability of zinc to affect the ionophore A23187 doseresponse curve indicates that the effect of zinc is not mediated through alterations in $\mathrm{Ca}^{2+}$ fluxes.

A shift in the dose response of $\mathrm{CB}$, as demonstrated, may also result if the uptake of CB is increased by zinc. However, in such an event the shift would be expected to occur at all three mitogen concentrations tested, but Table 3 shows that a clear shift occurs only at optimal concentration of the mitogen, a concentration where zinc exerts its specific differential effect as discussed above. Another mechanism one might consider is the effect $\mathrm{CB}$ may have on the transport of small molecules (23) and the possibility that zinc might affect the transport of these molecules in a synergistic manner. Although this possibility cannot be excluded we would like to place greater emphasis on the cytoskeletal system for several reasons: (a) one of the effects of CB on transport function is the enhancement of $\mathrm{Ca}^{2+}$ uptake (22) and our experiments show that zinc does not operate by this 


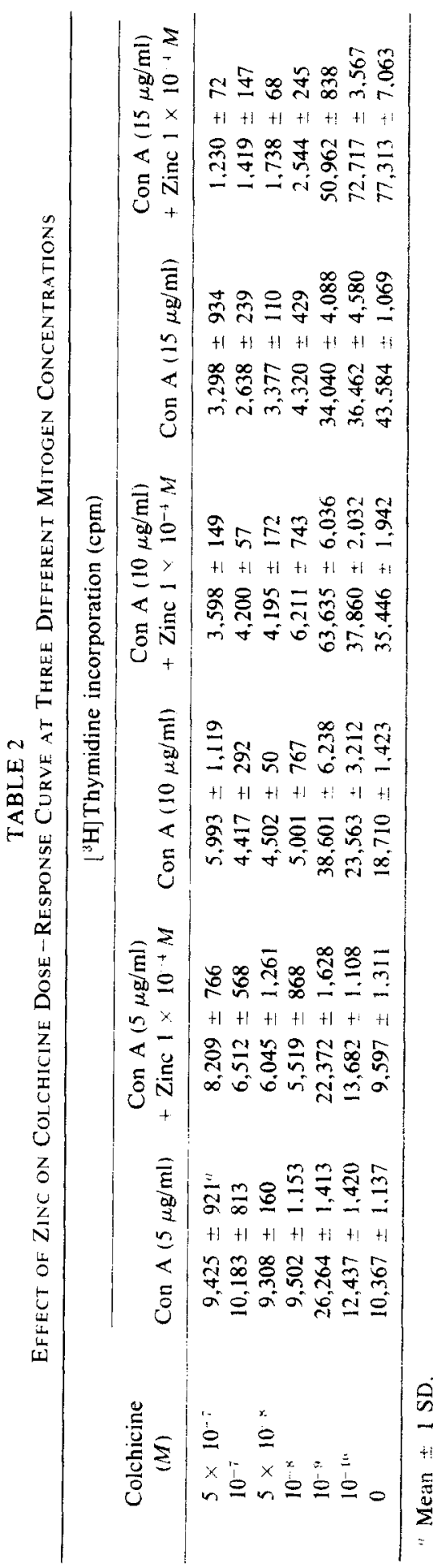




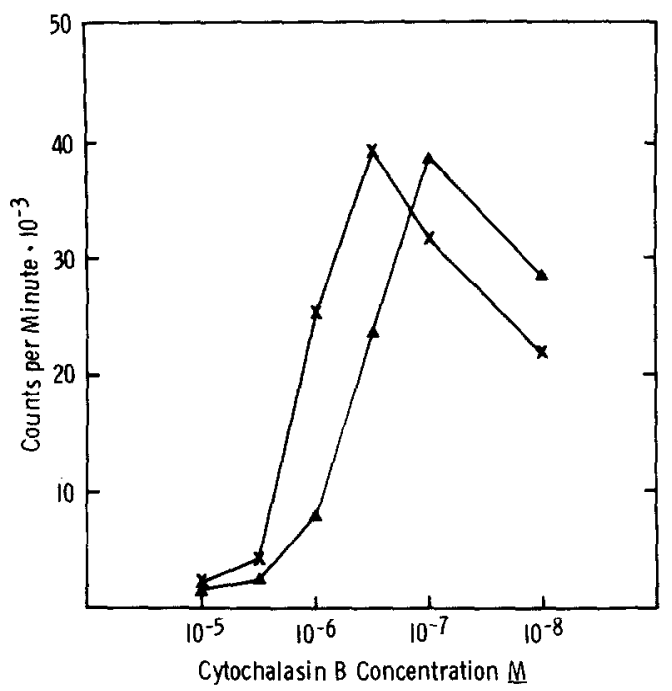

FIG. 5. Effect of zinc on the cytochalasin B dose-response curve. $\mathrm{x}$, Con A $(10 \mu \mathrm{g} / \mathrm{ml}) ; \boldsymbol{A}$, Con A $(10 \mu \mathrm{g} / \mathrm{ml})+\mathrm{Zn} 1 \times 10^{-4} M$.

mechanism, thus excluding at least one transport property as not being relevant to the shift we observe with zinc; (b) zinc has been demonstrated to have a direct effect on red cell membranes (24) and the red cell membrane has high-affinity binding sites for CB (23), thus precedence exists for suggesting that both these agents might be acting on the lymphocyte membrane through the cytoskeleton; (c) experiments in rats indicate that mobility of the surface membrane of lymphocytes is altered with age and it is postulated that this alteration might reflect changes in the cytoskeleton (25) (our findings are consistent with such a hypothesis); and finally (d) it is very likely that alterations in the transport of small molecules might be secondary to changes in the cytoskeletal function which are not perceptible by morphological studies.

Another intriguing finding which might support the similarity between the action of zinc and $C B$ is the observation that $C B$ inhibits rosette formation of sheep erythrocytes with thymus-derived peripheral blood lymphocytes but not with thymocytes (26). This observation is analogous to our finding that zinc has differential effects on lymphocytes obtained from young and aged individuals (11).

Several studies have suggested that membrane changes, reduction in the number of receptors, or changes in receptor sensitivity may be involved in the age-related decline of the immune response $(27,28)$. Similarly, receptor concentrations of various hormones were found to decrease with increasing age (29). Microfilaments have profound influence on membrane mobility, receptor expression, and sensitivity $(30,31)$. Thus any change in the structure and function of the microfilaments and related structures will have significant effects on all aforementioned parameters. Our previous observations that zinc has differential effects on lymphocytes obtained from young and aged individuals and the present evidence that zinc might act through microfilaments, actin, or similar structures point toward an area of research which might yield fruitful results in understanding 


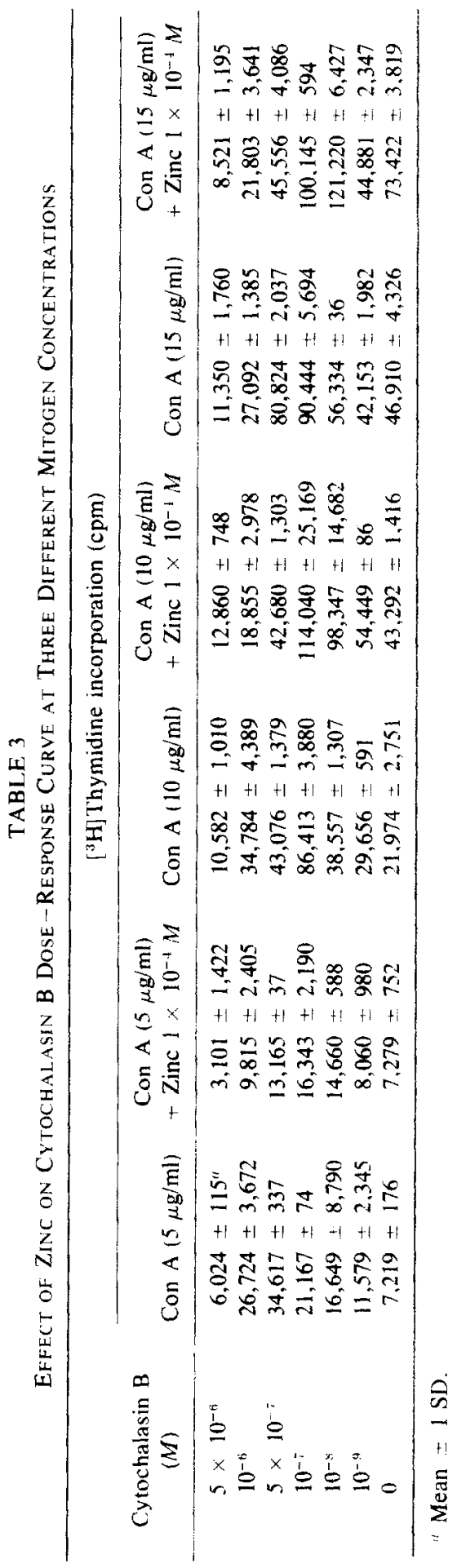


the mechanism of immunological senescence. If our future studies implicate changes in actin or similar elements in the depressed immunologic responses in aging, the existence of these elements in almost every cell in the organism offers an attractive unifying theory for the cellular basis of aging.

\section{ACKNOWLEDGMENTS}

This research was supported by American Cancer Society Grant IM-126A and the Children's Leukemia Foundation of Michigan. We wish to thank Dr. Robert L. Hamill, Eli Lilly Research Laboratories, Indianapolis, Indiana for generously supplying ionophore A23187 and nigericin.

\section{REFERENCES}

1. Kroneman, J., Van d. Mey, G. J. W., and Helder, A., Zentralbl. Veterinaermed., A 22, $201,1975$.

2. Tanaka, T., Fernandes, G., Tsao. C. Pih, K., and Good, R. A., Fed. Proc. 37, 931, 1978. (Abstract)

3. Iwata, T., Incefy, G. S., Tanaka, T., Fernandes, G., Menendez-Botet, C. J., Pih, K., and Good, R. A., Fed. Pror. 37, 1827, 1978. (Abstract)

4. Luecke, R. W., Simonel, C. E., and Fraker, P. J., J. Nutr. 108, 881, 1978.

5. Frost, P., Chen, J. C., Rabbani, I., Smith, J., and Prasad, A. S., In 'Zinc Metabolism: Current Aspects in Health and Disease" (G. J. Brewer and A. S. Prasad, Eds.), pp. 143-150. Liss, New York, 1977.

6. Tucker, S. B., Schroeter, A. L., Brown, P. W., and McCall, J. T., J. Amer. Med. Assoc. 235, $2399,1976$.

7. Oleske, J. M., Caleb, M. H., and Starr, S. E., Cutis 21, 297, 1978.

8. Moynahan, E. J., Lancet 2, 399, 1974.

9. Rühl, H., Kirchner, H., and Bochert, G., Proc. Soc. Exp. Biol. Med. 137, 1089, 1971.

10. Berger, N. A., and Skinner, A. M., J. C'ell. Biol. 61, 45, 1974.

11. Rao, K. M. K., Schwartz, S. A., and Good, R. A., Cell. Immunol. 42, $270,1979$.

12. Luckasen, J. R., White, J. G., and Kersey, J. H., Proc. Nat. Acad. Sci. USA 71, 5088, 1974.

13. Daniele, R. P., Holian, S. K., and Nowell, P. C., J. Exp. Med. 147, 571, 1978.

14. Medrano, E., Piras, R., and Mordoh, J., Exp. Cell. Res. 86, 295, 1974.

15. Greene, W. C., Parker, C. M., and Parker, Ch. W., Exp. Cell. Res. 103, 109, 1976.

16. Hoffman, R., Ferguson, R., and Simmons, R. L., J. Immunol, 118, 1472, 1977.

17. Wessells, N. K., Spooner, B. S., Ash, J. F., Bradley, M. O., Luduena, M. A., Taylor, E. L., Wrenn, J. T., and Yamada, K. M., Science 171, 135, 1971.

18. Böyum, A., Scand. J. Clin. Lab. Invest. 21 (Suppl. 97), 77, 1971.

19. Rao, K. M. K., Schwartz, S. A., and Good, R. A., Cell Immunol. 48, $155,1979$.

20. Holtzer, H., and Sanger, J. W., Develop. Biol. 27, 444, 1972.

21. Hartwig, J. H., and Stossel, T. P., J. Cell. Biol. 71, 295, 1976.

22. Henney, C. S., In "Cytochalasins, Biochemical and Cell Biological Aspects" (S. W. Tannenbaum, Ed.), pp. 191-215. Elsevier, Amsterdam, 1978.

23. Lin, S., and Spudich, J. A., J. Biol. Chem. 249, 5778, 1974.

24. Eaton, J. W., Berger, E., White, J. G., and Jacob, H. S., In "Zinc Metabolism: Current Aspects in Health and Disease" (G. J. Brewer and A. S. Prasad, Eds.), pp. 275-293. Liss, New York, 1977.

25. Woda, B. A., Yguerabide, J., and Feldman, J. D., J. Immunol. 123, 2161, 1979.

26. Radaszkiewicz, T., Kotz, D., and Denk, H., Clin. Exp. Immunol. 25, 501, 1976.

27. Meredith, P., Gerbase-DeLima, M., and Walford, R. L., Exp. Gerontol. 10, 247, 1975.

28. Price, G. B., and Makinodan, T., J. Immunol. 108, 403, 1972.

29. Roth, G. S., Fed. Proc. 38, 1910, 1979.

30. Edelman, G. M., Yahara, I., and Wang, J. L., Proc. Nat. Acad. Sci. USA 70, 1442, 1973.

31. Sundqvist, K.-G., and Fhrnst, A., Nature (London) 264, 226, 1976. 